\title{
Relationships between internal and external stability for infinite- dimensional systems with applications to a servo problem
}

$\operatorname{AUTHOR}(\mathrm{S}):$

YAMAMOTO, Y; HARA, S

\section{CITATION:}

YAMAMOTO, Y ... [et al]. Relationships between internal and external stability for infinitedimensional systems with applications to a servo problem. IEEE TRANSACTIONS ON AUTOMATIC CONTROL 1988, 33(11): 1044-1052

\section{ISSUE DATE:}

1988-11

URL:

http://hdl.handle.net/2433/50292

\section{RIGHT:}

(c) 1988 IEEE. Personal use of this material is permitted. However, permission to reprint/republish this material for advertising or promotional purposes or for creating new collective works for resale or redistribution to servers or lists, or to reuse any copyrighted component of this work in other works must be obtained from the IEEE. 


\title{
Relationships Between Internal and External Stability for Infinite-Dimensional Systems with Applications to a Servo Problem
}

\author{
YUTAKA YAMAMOTO, MEMBER, IEEE, AND SHINJI HARA, MEMBER, IEEE
}

\begin{abstract}
This paper studies the relationships among various stability notions for a class of infinite-dimensional systems, which contains a class of systems not covered by the existing method, e.g., those having infinitely many unstable poles. It is proved that: i) internal $L^{2}$-stability and exponential stability are equivalent; ii) internal stability implies $H^{\infty}$ stability. Several necessary and sufficient conditions for internal stability are derived. In particular, it is proved that, under certain conditions, a canonical realization is internally stable iff it is externally stable. These results are applied to the servo problem involving this class of systems. It is shown that: i) an internal model is necessary for tracking; ii) an internal model along with closed-loop stability implies tracking. A typical example, called a repetitive control system, is discussed to illustrate the results.
\end{abstract}

\section{INTRODUCTION}

$\mathrm{R}$

ECENT advances both in theory and technology require the solution of more and more advanced and nonclassical control problems. For example, there are varied practical demands to design a servo system which has the capability of tracking any periodic reference signal of a fixed period $L$. A recently introduced control scheme called repetitive control has been designed to meet this requirement. The scheme has in fact proved to be very effective for many applications: control of a proton synchrotron magnet power supply [16], an electric servomechanism [17], and robot manipulators [13], [18] are examples of actual applications of this scheme.

A typical repetitive control system takes the construction as shown in Fig. 1. The basic idea here is to incorporate an internal model $1 /\left(e^{L s}-1\right)$ into the system, where $L$ is the fixed period of reference signals, and then stabilize the closed-loop system. It is easy to observe that this internal model has the ability of producing any periodic signal with a suitable initial function. Hence, in view of the well-known internal model principle [11], the output is expected to track any periodic reference signal.

It should be, however, noted that this scheme raises several theoretical problems, which require a new theoretical challenge. First, the open-loop system becomes an infinite-dimensional system with infinitely many unstable poles. There exists, however, no servo theory for such systems at present. Indeed, servo systems for infinite-dimensional systems, in general, have not been studied extensively in the literature. The only exceptions are Francis [10] and Desoer and Gustafson [8]. However, these studies do not fit our requirements in the following ways.

1) Since they are based upon the $L^{2}$ input/output stability, the

Manuscript received August 31, 1987; revised March 15, 1988. This paper is based on a prior submission of January 27, 1987. Paper recommended by Associate Editor, T. J. Tarn.

Y. Yamamoto is with the Department of Applied Systems Science, Faculty of Engineering, Kyoto University, Kyoto, Japan.

$S$. Hara is with the Department of Control Engineering, Faculty of Engineering, Tokyo Institute of Technology, Tokyo, Japan.

IEEE Log Number 8823318.

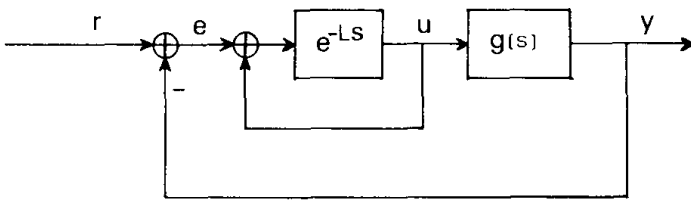

Fig. 1. Repetitive control system.

exponential decay of the error, which is what we really desire, is not necessarily guaranteed.

2) They cannot deal with systems with infinitely many unstable poles as is the case with repetitive control systems.

In view of these, we need a new framework that resolves these problems. The following questions must be taken into account.

1) What is the natural framework to study servo problems for infinite-dimensional systems containing the above class of systems?

2) Does the internal model principle carry over to such a class of systems? If so, in what form is it possible?

3) Should we take an external (input/output) approach or internal (state-space) approach to study these problems?

4) What are the relationships between the external and internal points of view? In particular, what can we say about stability from the external and internal points of view? Are they equivalent? If not, what are their inter-relationships?

Let us elaborate more upon these points. The two main objectives in servo theory are stability and tracking. There can be two approaches to attack these problems, one from the external (transfer function) viewpoint (e.g., [1]) and the other from the internal (state-space) one (e.g., [11]). Each has its own advantages. The external point of view generally leads to a more concise expression of the results, and its connection to the finitedimensional theory is more easily accessible. Thus, the extension to the infinite-dimensional case is easier to formulate and conjecture. For example, the internal model principle given in Section IV can be neatly expressed in the external form, whereas there is no such approach in the state-space form even in the case of delay-differential systems.

On the other hand, the external point of view generally presents a difficulty when dealing with stability. As noted above, what we really need in practice, especially in servo systems, is exponential stability rather than $L^{2}$ input/output stability. For example, one wants to conclude that the error $e(t)$ tends exponentially to zero. This leads us to the need to deal with internal stability rather than external stability, and the state-space approach becomes necessary. It also induces the question of whether or not the constructed scheme is irredundant, thereby not introducing an arbitrary element to the scheme.

In order to handle all these problems, it is necessary to establish a firm link between the external and internal approaches. For example, it is highly desirable to have an external condition that guarantees internal stability. This will also require the clarification of the notion of irredundant (canonical) realizations. Unfor- 
tunately, for infinite-dimensional systems, the relationship between internal and external stability has not been clarified in a sufficiently general setting.

To satisfy the above requirements, we shall make use of the class of pseudorational impulse responses studied in [27] and [28]. Roughly speaking, transfer functions in this class are of the form $Q^{-1}(s) P(s)$ where $Q(s)$ and $P(s)$ are entire (i.e., holomorphic on the whole $\boldsymbol{C}$-plane) complex functions of exponential type [27], [28] (hence, the transfer functions are always meromorphic), and hence are a very natural generalization of rational transfer matrices. Transfer functions of delay-differential systems are always of this type. On the contrary, the framework using the algebra $Q$ by Desoer and others ([3], [6]-[8], etc.) does not have this property. (With regard to the relationship of our framework and theirs, neither contains the other properly. However, for the above reason, the pseudorational class is more appropriate for our present purposes. For a more detailed discussion, see Section II.)

Our objective in this paper is twofold. One is to clarify the relationship between internal and external stability, and the other to study the servo problem, for the class of pseudorational impulse response matrices. The paper is organized as follows. After presenting basic materials of pseudorational impulse responses in Section II, we give the basic results on internal and external stability in Section III. There is no such theory at present in the context of infinite-dimensional systems, but that does not suggest that it is at all trivial. (Recently, Callier and Winkin [4] gave a frequency domain condition to guarantee exponential stability. However, their framework utilizes the algebra $Q$ [6] of stable impulse responses, and does not contain the case of infinitely many unstable poles.) It is proved that:

i) internal stability implies external stability;

ii) with additional conditions, external stability implies internal stability of the canonical realization.

Section IV applies these results to the analysis of the servo problem in the pseudorational class in general. The internal model principle will be proved; that is,

i) an internal model is necessary for tracking;

ii) an internal model plus closed-loop stability implies tracking.

As a consequence of stability results in Section III, we can conclude exponential decay of the error as desired. Section $\mathrm{V}$ is devoted to the application of these results to the repetitive control system along with a numerical example to illustrate the theory.

\section{Notation AND CONVENTION}

In what follows, various spaces of distributions will play crucial roles. We here introduce those spaces along with some of their basic properties. For details, see [20], [22], and [26], etc.

$D^{\prime}$ (respectively, $\left.D^{\prime}\right):=$ the space of distributions on $\boldsymbol{R}$ with support bounded on the left (respectively, right).

$\mathcal{E}^{\prime}(\boldsymbol{R})$ [respectively, $\left.\mathcal{E}^{\prime}\left(\boldsymbol{R}^{-}\right)\right]:=$the space of distributions on $\boldsymbol{R}$ with compact support (respectively, compact support contained in $(-\infty, 0])$.

All these spaces each constitute a convolution algebra with identity $\delta$ (the Dirac delta distribution at the origin). The delta distribution at point $a$ will be denoted by $\delta_{a}$, and its derivative will be denoted by $\delta_{a}^{\prime}$. Convolution will be denoted by $*$, as usual.

Let $\mathcal{D}[0, \infty)$ denote the space of $C^{\infty}$-functions defined on ($\infty, \infty)$ having compact support contained in $[0, \infty)$, and denote by $D^{\prime}[0, \infty)$ its dual space. By considering the dual of the canonical injection $j$, which embeds $D[0, \infty)$ into the space of $C^{\infty}$-functions with support bounded on the right, we obtain the canonical projection

$$
\pi: \mathfrak{D}_{+}^{\prime} \rightarrow \mathbb{D}^{\prime}[0, \infty)
$$

which is an extension of the truncation $\pi \varphi:=\left.\varphi\right|_{[0, \infty)}$.

Given a distribution $\alpha$, its support is denoted by supp $\alpha$. When $\alpha$ is a vector or a matrix with distribution entries, supp $\alpha$ is understood to be the union of the supports of all its entries.

For a distribution $\alpha \in D_{-}^{\prime}, r(\alpha)$ denotes the least upper bound of supp $\alpha$ :

$$
r(\psi):=\sup \{t \in \operatorname{supp} \psi\} .
$$

Concerning $r(\varphi * \psi)$, the following identity holds which is a consequence of the well-known theorem of Titchmarsh on convolution $[9$, p. 224]:

$$
r(\varphi * \psi)=r(\varphi)+r(\psi) .
$$

The space $\Omega^{m}:=\left(U_{n>0} L^{2}[-n, 0]\right)^{m}$, with the inductive limit topology (see [22], [25]), is called the space of inputs, and $\Gamma^{p}$ $:=\left(L_{\mathrm{loc}}^{2}[0, \infty)\right)^{p}$, i.e., the $p$-product of the space of locally Lebesgue square integrable functions on $[0, \infty)$, is called the space of outputs. For a locally square integrable function $\psi$, $\|\psi\|_{[a, b]}$ denotes its $L^{2}$-norm on $[a, b]$. On the other hand, $\|\psi\|_{2}$ denotes the $L^{2}$-norm of $\psi \in L^{2}[0, \infty)$. These spaces are each equipped with a left shift semigroup $\sigma_{t}$ (denoted for convenience by the same symbol) defined as follows:

$$
\begin{aligned}
\left(\sigma_{t} \omega\right)(\tau):= & \omega(\tau+t), \tau \leq-t, 0,-t \leq \tau \leq 0, \omega \in \Omega^{m} ; \\
& \left(\sigma_{t} \gamma\right)(\tau):=\gamma(\tau+t), \gamma \in \Gamma^{p} .
\end{aligned}
$$

In what follows, we shall often need to deal with matrices with distribution entries. It often makes the statement very cumbersome to write $Q \in\left(\mathcal{E}^{\prime}\left(\boldsymbol{R}^{-}\right)\right)^{p \times m}$, etc., in which case, we shall often abbreviate it as $Q \in \mathcal{E}^{\prime}\left(R^{-}\right)$, meaning, of course, that each entry of $Q$ belongs to $\varepsilon^{\prime}\left(\boldsymbol{R}^{-}\right)$.

We denote by $J$ the following subset of $\mathcal{E}^{\prime}\left(\boldsymbol{R}^{-}\right)$:

$$
J:=\left\{\varphi \in \mathcal{E}^{\prime}\left(\boldsymbol{R}^{-}\right) ; r(\varphi)<0\right\} .
$$

In view of (1.3), $J$ is a prime ideal of $\mathcal{E}^{\prime}\left(\boldsymbol{R}^{-}\right)$, and hence the quotient ring $\mathcal{E}^{\prime}\left(\boldsymbol{R}^{-}\right) / J$ is an integral domain. We denote by $\mathcal{F}$ the field of fractions consisting of elements of $\varepsilon^{\prime}\left(R^{-}\right) / J$.

The (bilateral) Laplace transform of a distribution $\alpha$ will be denoted by $\hat{\alpha}(s)$ or by $\mathscr{L}[\alpha](s)$.

As usual, $H^{\infty}$ denotes the space of holomorphic functions on the open right-half plane which possess nontangential limits to the imaginary axis almost everywhere and this boundary function is essentially bounded. For a matrix $A$ over $H^{\infty}$, its $H^{\infty}$-norm $\|A\|_{\infty}$ is the supremum of the greatest singular value of $A(j \omega)$ :

$$
\|A\|_{\infty}:=\sup _{-\infty<\omega<\infty} \sigma_{\max }(A(j \omega)) .
$$

The closed right-half plane will be denoted by $C^{+}$.

\section{Preliminaries: Summary of PSeudorational ImPUlSe RESPONSES}

In what follows we shall exclusively deal with those impulse response matrices having a particular type of fractional representation. We call such impulse responses pseudorational. To introduce the notion of pseudorationality, we first make the following definition.

Definition 2.1: Let $Q$ be a square matrix with entries in $\mathcal{E}^{\prime}\left(R^{-}\right) . Q$ is said to be of normal type if the following conditions are satisfied:

i) det $Q$ is invertible with respect to convolution over $\mathcal{D}_{+}^{\prime}$, and supp $Q^{-1} \subset[0, \infty)$;

ii) ord $(\operatorname{det} Q)^{-1}=-$ ord $(\operatorname{det} Q)$,

where ord $\alpha$ denotes the order of a distribution $\alpha$ [20].

A $p \times m$ matrix with entries which are (Radon) measures on $[0, \infty)$ (see [25] for details) is called an impulse response matrix. An impulse response matrix $A$ induces a (zero initial state) inputoutput correspondence as follows:

$$
f(u)=\pi(A * u), u \in \Omega^{m} .
$$


Here $\pi$ is the projection defined by (1.1), $u \in \Omega^{m}$ is an input vector, and the output vector $f(u)$ is understood to belong to the space $\Gamma^{p}$. Note that when the input $u$ is given on $[0, t)$ and if the entries of $A$ are ordinary functions, then (2.2) is converted to the more familiar form

$$
f(u)(t)=\int_{0}^{t} A(t-\tau) u(\tau) d \tau .
$$

The pseudorational impulse responses are now introduced as follows.

Definition 2.3: Let $A$ be an impulse response matrix. $A$ is said to be pseudorational if it can be written in the form

$$
A=Q^{-1} * P
$$

for some matrices $Q$ and $P$ over $\mathcal{E}^{\prime}\left(R^{-}\right)$where the $p \times p$ matrix $Q$ is of normal type.

As pointed out in the Introduction, not all impulse responses are pseudorational. However, the important class of impulse responses of delay-differential systems (retarded or neutral) are known to be pseudorational [27], [29]. Let us here make a few remarks on the comparison of our framework with the framework using the algebra $Q$ of stable impulse responses by Desoer and others [3], [6], [7], [24]. In these approaches, some infinitedimensional servo problems have been studied from the external point of view [8], [10]. The algebra $a$ consists of those impulse responses having the expression $f+\Sigma_{i} a_{i} \delta\left(t-t_{i}\right), f \in L^{1}[0$ $\infty), \Sigma\left|a_{i}\right|<\infty$. Because of an invertibility requirement over $\mathbb{A}$, their framework does not admit infinitely many unstable poles [7], [8]. This restricts the applicability, especially to the repetitive control system in Section I. On the other hand, while the Laplace transform of a pseudorational impulse response is always meromorphic as pointed out in Section $\mathrm{I}$, not all $L^{1}$-functions have Laplace transforms meromorphic on the entire complex plane, so that certain elements in $\mathcal{Q}$ do not belong to our framework. Hence, neither framework contains the other. However, in view of the problem of infinitely many unstable poles and the problem of guaranteeing internal stability by an external condition, the pseudorational class is more appropriate for our present purposes.

It is known [26], [27] that for every pseudorational impulse response, we can construct a standard observable realization as follows.

Let $A=Q^{-1} * P$ be pseudorational. Let $X^{Q}$ be a subspace of $\Gamma^{p}$ defined by

$$
X^{Q}:=\left\{x(t) \in \Gamma^{p} ; \pi(Q * x)=0\right\}
$$

$X^{Q}$ is easily seen to be a closed subspace of $\Gamma^{p}$. Moreover, it is isomorphic to a Hilbert space [26], [27]. Roughly speaking, the standard topologically observable realization we shall be concerned with is constructed as follows [26], [27].

i) State space $:=X^{Q}$.

ii) The state $\varphi(t, x, u)$ at time $t$ resulting from an input $u$ and the initial state $x$ is given by

$$
\varphi(t, x, u):=\sigma_{t} x+\pi\left(A * \sigma_{t}^{Q} u\right), x \in X^{Q},
$$

where $\left(\sigma_{t}^{\Omega} u\right)(s):=u(s+t)$. (This formula is obtained by suitably shifting the input function and using the shift-invariance.)

iii) The output $y(t)$ of the system is given by $y(t):=x_{t}(0)$, $x_{t}(\cdot) \in X^{Q}$

We denote this system by $\Sigma^{Q, P} ; \Sigma^{Q}$ stands for $\Sigma^{Q, I}$. The following facts will be used in the sequel.

Facts 2.6 [26]-[28]:

a) There exists $T>0$ such that $X^{Q}$ is topologically isomorphic to its restriction $\left.X^{Q}\right|_{[0, T]}$ to the interval $[0, T]$ where the latter is endowed with the subspace topology induced from $L^{2}[0, T]$. In what follows, the norm of $X^{Q}$ will be understood to be $\|x\|_{[0, T]}$ for one of such $T>0$.

b) The system $\Sigma^{Q, P}$ is quasi-reachable (i.e., the reachable space is dense) if and only if the pair $Q$ and $P$ are approximately left coprime over $\mathcal{E}^{\prime}\left(\boldsymbol{R}^{-}\right)$[26], i.e., there exists a sequence of matrices $R_{n}$ and $S_{n}$ over $\mathcal{E}^{\prime}\left(R^{-}\right)$of suitable sizes such that

$$
Q * R_{n}+P * S_{n} \rightarrow \delta I \text {. }
$$

Since $\Sigma{ }^{Q, P}$ is always topologically observable, this is equivalent to the canonicity of $\Sigma Q, P$.

c) The spectrum $\sigma(F)$ of the infinitesimal generator of the shift semigroup $\left\{\sigma_{t}\right\}$ is given by

$$
\sigma(F)=\{\lambda \in C ; \operatorname{det} \hat{Q}(\lambda)=0\} .
$$

d) $X^{D} \subset X^{Q}$ if and only if $Q=M * D$ for some matrix $M$ with entries in $\mathcal{E}^{\prime}\left(\boldsymbol{R}^{-}\right)$.

e) According to the Paley-Wiener theorem for distributions [20], [22], [26], the Laplace transform $\hat{\alpha}(s)$ of $\alpha \in \mathcal{E}^{\prime}\left(\boldsymbol{R}^{-}\right)$is an entire function of $s$ satisfying the following estimate:

$$
\begin{aligned}
|\hat{\alpha}(s)| & \leq C(1+|s|)^{m} \exp (a \operatorname{Re} s), \operatorname{Re} s \geq 0, \\
& \leq C(1+|s|)^{m}, \operatorname{Re} s<0 .
\end{aligned}
$$

Hence, the Laplace transform of each element of $Q$ and $P$ is an entire function satisfying the above estimate.

\section{INTERnAL AND EXTERnal STABILITY}

In this section we shall establish interrelationships among various stability notions for the standard (topologically) observable realization $\Sigma^{Q, P}$ introduced in the previous section. We shall prove that:

i) internal $L^{2}$-stability and exponential stability are equivalent;

ii) internal $L^{2}$-stability implies external $H^{\infty}$-stability, and give necessary and sufficient conditions for internal stability.

Unlike the finite-dimensional case, such results are not trivial at all. To elaborate, consider the impulse response $A(t)=e^{-t} \sin$ $e^{t}$. This impulse response maps $z$ inputs to $L^{2}$ outputs, so that one may conclude that this impulse response is externally stable. However, it does not induce an exponentially stable canonical realization. To see this, consider the derivative $A^{\prime}=-e^{-t} \sin e^{t}$ $+\cos e^{t}$, which does not belong to $L^{2}$. By choosing a suitable $L^{2}$ input, one can produce a free response which is arbitrarily close to $A^{\prime} \notin L^{2}$. Since this kind of phenomenon would not occur if the corresponding realization were exponentially stable, one must conclude that the above $A$ does not induce an internally stable canonical realization.

Some necessary and sufficient conditions for internal stability are also derived.

Definitions 3.1: Let $\Sigma^{Q, P}$ be the pseudorational system associated with a pseudorational impulse response $A=Q^{-1} * P$. $\Sigma{ }^{Q, P}$ is said to be exponentially stable if there exist constants $M$, $\beta>0$ such that

$$
\left\|\sigma_{t} x\right\| \leq M \exp (-\beta t)\|x\|, \quad \text { for all } t \geq 0 \text { and } x \in X^{Q}
$$

where the norm $\|x\|$ is taken in the sense of Fact 2.6a).

$\Sigma^{Q, P}$ is said to be internally $L^{2}$-stable if

$$
X^{Q} \subset L^{2}[0, \infty)
$$

Definition 3.3: Let $\Sigma^{Q, P}$ be as above. $\Sigma^{Q, P}$ is said to be $H^{\infty}$. stable if the Laplace transform $\hat{A}(s)$ of $A$ belongs to $H^{\infty}$.

Remark 3.4: By the definition of the space $H^{\infty}, \dot{\hat{A}}(s)$ is analytic on the open right-half plane. However, since $\hat{A}(s)=$ $\hat{Q}(s)^{-1} \hat{P}(s)$ where each entry of $\hat{Q}(s)$ and $\hat{P}(s)$ is an entire function of $s$ [Fact 2.6e)], any singularity of $\hat{A}(s)$ must be a pole, and this pole cannot lie on the imaginary axis if $\hat{A}(s)$ belongs to $H^{\infty}$. Therefore, the above condition implies that $\hat{A}(s)$ is holomorphic on the closed right-half plane $C^{+}$. 
We first prove the equivalence of $L^{2}$-internal stability and exponential stability.

Theorem 3.5: Let $\Sigma^{Q}$ and $\Sigma^{Q, P}$ be as above. The following statements are equivalent:

i) $\Sigma^{Q}$ is internally $L^{2}$-stable;

ii) $\sum^{Q, P}$ is internally $L^{2}$-stable;

iii) $\Sigma Q, P$ is exponentially stable.

Proof: Since the state-space $X^{Q}$ is common to all $\Sigma^{Q, P}$ with the same $Q$, i) and ii) are clearly equivalent. It is also obvious that iii) implies ii). It remains only to prove ii) $\Rightarrow$ iii). We claim that, for every $x \in X^{Q}$,

$$
\int_{0}^{\infty}\left\|\sigma_{t} x\right\|^{2} d t<\infty
$$

Recall that the norm of $X^{Q}$ is given by the $L^{2}$-norm on some bounded interval $[0, T]$ [Fact 2.6a)]. Since each $x$ is an element of $L^{2}[0, \infty)$, the correspondence

$$
\tau \mapsto\left\|\sigma_{\tau} x\right\|^{2}
$$

is a continuous function of $\tau$ so that this is integrable on $[0, T]$. Thus, we have

$$
\int_{0}^{T} d \tau \int_{0}^{\infty}|x(\tau+t)|^{2} d t<\infty
$$

which implies, by Fubini's theorem, that

$$
\int_{0}^{\infty} d t \int_{0}^{T}|x(\tau+t)|^{2} d \tau=\int_{0}^{\infty}\left\|\sigma_{t} x\right\|^{2} d t<\infty
$$

Now according to a result due to Datko, any strongly continuous semigroup $\sigma_{t}$ that satisfies the property (3.6) is exponentially stable [5, last Corollary]. Therefore, $\Sigma Q$ is exponentially stable. $\square$

Remark 3.7: In view of the equivalence ii) $\Leftrightarrow$ iii), we may simply say, without ambiguity, that a system $\Sigma Q, P$ is internally stable. Furthermore, by virtue of the equivalence i) $\Leftrightarrow$ ii), we may speak of the internal stability of systems $\Sigma Q, P$, independently of each $P$. Henceforth, we may simply state that the system $\Sigma^{Q}$ is internally stable, meaning implicitly that all $\Sigma Q, P$ are simultaneously internally stable.

Corollary 3.8: Let $A$ be a pseudorational impulse response such that it admits an approximately left coprime fractional representation $A=Q^{-1} * P$. Then the canonical realization of $A$ is exponentially stable if and only if $\Sigma^{Q}$ is internally stable.

Proof: Obvious from the above theorem since the canonical realization of $A$ is $\Sigma Q, P$ (Fact 2.6).

The following theorem asserts that the system $\Sigma Q, P$ is externally stable if it is internally stable. Although it appears to be trivially true, the proof is surprisingly involved; this is because we must also consider the case in which the impulse response is not a function but merely a measure. Such a case must be included into the framework to deal with systems studied in Section V.

Theorem 3.9: Suppose that $\Sigma{ }^{Q, P}$ is internally stable. Then it is $H^{\infty}$-stable. Furthermore, the statement remains valid for any pseudorational $A_{1}=Q^{-1} * P_{1}$. In particular, det $\hat{Q}(s)^{-1}$ $\in H^{\infty}$.

Proof: See the Appendix.

Corollary 3.10: Consider the scalar case, i.e., we assume that the number of output channels $p=1$. Write $q$ instead of $Q$ and let $r:=$ ord $q$. Suppose that $\Sigma^{q}$ is internally stable. Then

$$
\sup _{\omega}\left|\frac{\omega^{r}}{\hat{q}(j \omega)}\right| \leq M .
$$

Proof. Observe that $q^{-1} *\left\{\delta^{\prime}\right\}^{r}$ is pseudorational, since ord $q=r$. Then the result follows from Theorem 3.9.

The converse of the above corollary also holds. Namely, we have the following.
Theorem 3.12: Under the same notation as in Corollary 3.10 , the system $\Sigma^{a}$ is internally stable if and only if

i) $\hat{q}^{-1}(s)$ belongs to $H^{\infty}$, and

ii) $\frac{1}{|\hat{q}(j \omega)|}=0\left(\frac{1}{|\omega|^{r}}\right)$, as $|\omega| \rightarrow \infty$.

Proof: We need only to show the sufficiency. Since $\hat{q}^{-1}(s)$ belongs to $H^{\infty}$, it is of exponential type on the open right-half plane, and hence so is $s^{r} / \hat{q}(s)$. Now according to a version of the Phragmén-Lindelöf principle [2, Theorem 6.2.4], every function that is holomorphic on the right-half plane, of exponential type there and bounded on the imaginary axis, is bounded by $C \exp$ $(a|\operatorname{Re} s|)$ on the right-half plane for some $C>0$ and $a \geq 0$. Therefore, we have

$$
\frac{\left|s^{r}\right|}{|\hat{q}(s)|} \leq C \exp (a|\operatorname{Re} s|), \quad a \geq 0, \operatorname{Re} s \geq 0 .
$$

Therefore, $s^{r} / \hat{q}(s) \exp (a s)$ belongs to $H^{\infty}$. Now consider the following function $\rho$ :

$$
\rho(t):= \begin{cases}\exp (\alpha(t+1)), & \text { for }-1 \leq t \leq 0, \\ 0, & \text { otherwise }\end{cases}
$$

where $\alpha:=\log (1 / 2)<0$. It is easy to see that $\rho=\left\{\delta_{-1}-\delta /\right.$ $2\} *\left\{\delta^{\prime}-\alpha \delta\right\}^{-1}$, and hence $\mathcal{L}[\rho]=\left\{e^{s}-1 / 2\right\} /(s-\alpha)$. Its inverse, with respect to convolution, is then computed as

$$
\rho^{-1}=\left\{\delta^{\prime}+\alpha \delta\right\} *\left\{\sum_{n=1}^{\infty}(1 / 2)^{n-1} \delta_{n}\right\}
$$

which acts as a first-order differential operator with respect to convolution. Therefore, in view of ord $q=r$, we see that both $\delta_{-a} * \rho^{r} * q$ and $\delta_{a} *\left\{\rho^{-1}\right\}^{r} * q^{-1}$ are distributions of order 0 , i.e., measures. Now put $q_{1}:=\delta_{-a} * \rho^{r} * q$. Then $q_{1}$ is of normal type, and satisfies $\mathscr{L}\left[q_{1}^{-1}\right] \in H^{\infty}$, by hypothesis. Hence, the reachable elements in $X^{q_{1}}$ all belong to $L^{2}[0, \infty)$. Showing that every element in $X^{q_{1}}$ is reachable will complete the proof. Take any $x$ in $X^{q_{1}}$, and put

$$
u:=q_{1} * x .
$$

(Here $x$ is extended to $(-\infty, \infty)$ by setting it zero on $(-\infty, 0)$.) Since $q_{1}$ is a measure, $u$ is a locally $L^{2}$ function. Also, its support is bounded on the left because $q_{1}$ and $x$ have the same property. Furthermore, since $x$ belongs to $X^{q_{1}}, \pi\left(q_{1} * x\right)=0$ by definition, and hence supp $q_{1} * x \subset(-\infty, 0]$. Therefore, $u$ is a legitimate input function belonging to $\Omega$. It now follows that

$$
x=\pi x=\pi\left(q_{1}^{-1} * q_{1} * x\right)=\pi\left(q_{1}^{-1} * u\right)
$$

so that every element in $X^{q_{1}}$ is reachable, and hence $X^{q_{1}} \subset L^{2}[0$, $\infty)$.

For the multivariable case, the condition (3.13) gives a sufficient condition for internal stability.

Theorem 3.17: The system $\Sigma^{Q}$ (and hence any $\Sigma^{Q, P}$ ) is internally stable if the condition (3.13) is satisfied for $q:=$ det $Q$.

Proof: Since $(\operatorname{det} Q) I=(\operatorname{adj} Q) * Q$, the space $X^{Q}$ is contained in $X^{q} \times X^{q} \times \cdots \times X^{q}(p$ times) by Fact 2.6. Since the semigroup $\sigma_{t}$ in $X^{q}$ is exponentially stable by Theorem 3.12 , so is $\sigma_{t}$ in $X^{Q}$

The following corollary is immediate from Theorem 3.12 and Fact 2.6.

Corollary 3.18: Consider the scalar case $p=1$. Let $A$ be $a$ pseudorational impulse response such that it admits an approximately corprime fractional representation $A=$ $q^{-1} * P$. Then its canonical realization is internally stable if and only if (3.13) holds. 


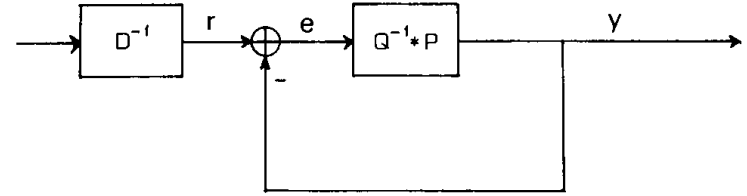

Fig. 2. Unity feedback servo system.

\section{Tracking and the Internal Model Principle}

In this section, we formulate and prove the internal model principle for the class of pseudorational impulse response matrices; which is a precise analog and generalization of the wellknown finite-dimensional counterpart [1], [11].

\section{Problem Statement}

Consider the unity-feedback servo system given in Fig. 2, where $D, Q, P$ are $p \times p$ matrices over $\mathcal{E}^{\prime}\left(\boldsymbol{R}^{-}\right)$satisfying the conditions of pseudorationality. Find a condition under which the error $e(t)$ converges to zero for any reference signal generated by $D^{-1}$. tion.

Moreover, it is assumed that $D$ satisfies the following assump-

Assumption 4.1: $D[$ or $\hat{D}(s)]$ is purely unstable in the sense that all poles of det $D(s)^{-1}$ lie in the (closed) right-half plane $C^{+}$ and $r(\operatorname{det} D)=0$, i.e., $D$ has no redundant delay part.

Remark 4.2: This assumption is not overly restrictive in the following sense: stable modes are clearly irrelevant to the asymptotic tracking. Secondly, according to a result of [28], the condition $r(\operatorname{det} D)=0$ corresponds to the nonexistence of a small solution, namely a function that becomes zero after a finite duration of time. Such functions in $X^{D}$ clearly do not affect the asymptotic tracking property, and hence are redundant for discussing tracking. If $r(\operatorname{det} D) \neq 0$ but $r(\operatorname{det} D)=r\left(d_{1}\right)+\cdots$ $+r\left(d_{p}\right)\left(d_{i}:=i\right.$ th row of $\left.D\right)$, then we can easily reduce $D$ to $D_{0}$ such that $r\left(\right.$ det $\left.D_{0}\right)=0$ by left-multiplying $D$ with a matrix consisting of elements of type $\delta_{-a}$ and 0 .

The objective in this section is to clarify the relationships between the tracking property and the internal model under the assumption of (4.1) and the closed-loop stability.

The following relationships hold among $r_{0}, r, e, y$ :

$$
\begin{aligned}
& y=\pi\left\{(Q+P)^{-1} * P * D^{-1} r_{0}\right\} ; \\
& e=\pi\left\{(Q+P)^{-1} * Q * D^{-1} r_{0}\right\} .
\end{aligned}
$$

Hence, tracking is implied either by

$$
\mathscr{L}\left[(Q+P)^{-1} * Q * D^{-1}\right](s) \text { belongs to } H^{\infty}
$$

or by

$e(t)$ tends exponentially to zero for any initial state in the reference signal generator

in the external or internal description sense, respectively. On the other hand, since the existence of an $M \in \mathcal{E}^{\prime}\left(R^{-}\right)^{p \times p}$ satisfying $Q=M * D$ implies $X^{D} \subset X^{Q}$ by Fact $\left.2.6 \mathrm{~d}\right)$, which means that every reference signal generated by $D^{-1}$ must be generated by $Q^{-1}$, it is reasonable to define the meaning of an internal model as follows.

Definition 4.6: $Q \in \mathcal{E}^{\prime}\left(\boldsymbol{R}^{-}\right)^{p \times p}$ is said to contain $D \in$ $\mathcal{E}^{\prime}\left(\boldsymbol{R}^{-}\right)^{p \times p}$ as an internal model if there exists $M \in \mathcal{E}^{\prime}\left(\boldsymbol{R}^{-}\right)^{p \times p}$ such that $Q=M * D$.

We first prove the necessity of an internal model for stable tracking in the following sense.

Theorem 4.7: Consider the unity feedback system Fig. 2. Suppose that

i) $(\hat{Q}+\hat{P})(s)^{-1} \hat{Q}(s) \hat{D}(s)^{-1}$ belongs to $H^{\infty}$;

ii) $\hat{D}(s)$ is purely unstable.
Then, there exists $M \in \mathcal{E}^{\prime}\left(\boldsymbol{R}^{-}\right)^{p \times p}$ such that $Q=M * D$. Hence, stable tracking implies the necessity of an internal model.

Proof: Since $(\hat{Q}+\hat{P})(s)^{-1} \hat{Q}(s) \hat{D}(s)^{-1}$ belongs to $H^{\infty}$, it is holomorphic on $C^{+}$. Moreover, since $(\hat{Q}+\hat{P})(s)$ is holomorphic on $C^{+}$, this implies that $\hat{Q}(s) \hat{D}(s)^{-1}$ is holomorphic on $C^{+}$. On the other hand, it is assumed that the zeros of $\operatorname{det} D(s)$ all belong to $C^{+}$. Hence, $\hat{Q}(s) \hat{D}(s)^{-1}$ must be an entire function of $s$. According to the Paley-Wiener-Schwartz theorem Fact $2.6 \mathrm{e})$, det $\hat{D}(s)$ is an entire function of exponential type, and hence so is $\hat{Q}(s) \hat{D}(s)^{-1}[28$, Lemma (A.1)]. Furthermore, since $\hat{Q}(s) \hat{D}(s)^{-1}$ is clearly inverse Laplace transformable, it follows from [28, Lemma (3.8)] that $Q * D^{-1}$ belongs to $\left(\mathcal{E}^{\prime}(R)\right)^{p \times p}$; that is, the space of distributions with compact support not necessarily contained in $(-\infty, 0]$. It remains only to prove that the support of $Q * D^{-1}$ is in fact contained in $(-\infty, 0]$.

Let $i: \mathcal{E}^{\prime}\left(\boldsymbol{R}^{-}\right) \rightarrow \mathcal{F}$ be the natural embedding of $\mathcal{E}^{\prime}\left(\boldsymbol{R}^{-}\right)$into the field $\mathcal{F}$ defined in Section $I$. This is clearly an algebra homomorphism. Since $r(\operatorname{det} D)=0, i(D)$ is of full rank. Suppose that the support of $Q * D^{-1}$ is not contained in $(-\infty, 0]$. Define $a$ by

$$
a:=\min \left\{b \geq 0 ; \delta_{-b} * Q * D^{-1} \in \mathcal{E}^{\prime}\left(\boldsymbol{R}^{-}\right)\right\} .
$$

By assumption, $a$ is positive. The identity

$$
\delta_{-a} * Q=\left(\delta_{-a} * Q * D^{-1}\right) * D
$$

yields the identity

$$
i\left(\delta_{-a} * Q\right)=i\left(\delta_{-a} * \mathrm{Q} * D^{-1}\right) * i(D)
$$

over $\mathcal{F}$, where $i\left(\delta_{-a^{*}} * Q\right)$ is clearly zero because $Q \in \mathcal{E}^{\prime}\left(\boldsymbol{R}^{-}\right)$and $a>0$. Furthermore, by the definition of $a, i\left(\delta_{-a^{*}} Q * D^{-1}\right)$ must be nonzero, for otherwise there would be a smaller $a>0$. But this is a contradiction because $i(D)$ is of full rank.

The necessity of an internal model in the sense of internal stability can be stated as follows.

Corollary 4.9: Suppose that:

i) error e $(t)$ tends exponentially to zero in the sense of (3.I) for any initial state in the reference signal generator; and

ii) $D$ is purely unstable. Then the conclusion of Theorem 4.7 remains valid.

Proof: Writing $D^{-1}$ as $(\operatorname{det} D)^{-1} *(\operatorname{adj} D)$, we see that $(Q+$ $P)^{-1} * Q * D^{-1}$ is pseudorational. By hypothesis $\mathrm{i}$ ), we see that the same argument in the proof of Theorem 3.9 applies to $(Q+$ $P)^{-1} * Q * D^{-1}$ with no change, and hence $(\hat{Q}(s)+$ $\hat{P}(s))^{-1} \hat{Q}(s) \hat{D}(s)^{-1}$ must belong to $H^{\infty}$. The result then follows from Theorem 4.7 .

Let us now prove the converse of Theorem 4.7. In view of the preceding corollary, we state the result in the form: under the hypothesis of internal stability, the existence of an internal model is equivalent to stable tracking.

Theorem 4.10: Consider the unity feedback system Fig. 2, and suppose that the closed-loop system $\Sigma^{(Q+P), P}$ is internally stable. Then the error $e(t)$ tends exponentially to zero Iin the sense of (3.1)] for any reference signal $r$ generated by $\Sigma^{D}$, if and only if $Q=M * D$ for some $M \in \mathcal{E}^{\prime}\left(\boldsymbol{R}^{-}\right)$, i.e., the forward-path of this system contains an internal model of $\Sigma^{D}$.

Proof: The necessity is Corollary 4.9 . Now observe that

$$
\begin{aligned}
e & =(Q+P)^{-1} * Q * r=(Q+P)^{-1} * Q * D^{-1} * u_{0} \\
& =(Q+P)^{-1} * M * u_{0}
\end{aligned}
$$

where $u_{0}:=D * r$. Since the system $\Sigma^{(Q+P), P}$ is exponentially stable, it follows from Theorem 3.4 that the system $\Sigma^{(Q+P)}$ is exponentially stable, and hence so is $\Sigma^{(Q+P), M}$. Therefore, the error converges exponentially to zero.

Observe that, as a result of dealing with internal stability, the above theorem assures exponential decay of the error $e(t)$ for any 

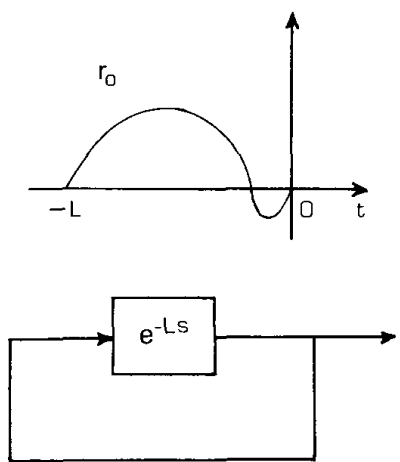

Fig. 3. Periodic signal generator and initial function.

initial state, in contrast to the results of the existing investigations (e.g., [8], [10]) given in terms of $H^{\infty}$-external stability. Note also that Theorem 3.17 gives an external condition guaranteeing internal stability needed here.

\section{ApPlication to Repetitive Control Systems}

In practice, one often encounters the situation in which the reference commands to be tracked and/or the disturbance to be rejected are periodic signals; repetitive commands or operations to industrial robots and $\mathrm{NC}$ machines, or disturbances depending on the frequency of the power supply are examples of such situations. A new control scheme named "repetitive control"' is introduced to deal with such situations and has proved to be very effective [13], [16]-[18]. Highly accurate asymptotic tracking can be achieved by implementing a periodic signal generator into this scheme. It is easily verified that any periodic signal with period $L$ can be generated by the linear input-free dynamical system shown in Fig. 3, where the waveform is specified by an initial function $r_{0}$. In other words, the transfer function of the periodic signal generator of period $L$ is given by

$$
\hat{D}(s)^{-1}=(\exp (L s)-1)^{-1},
$$

that is,

$$
D=\delta_{-L}-\delta .
$$

Let us first show that $D$ or $\hat{D}(s)$ defined by (5.2) or (5.1) satisfies the assumption in Theorem 4.7. Clearly, $D \in \mathcal{E}^{\prime}\left(\boldsymbol{R}^{-}\right)$, and $r(\operatorname{det} D)=0$ (i.e., $D$ is of full rank over the field $\mathfrak{F}$ ) since supp $D=\{0\} \cup\{-L\}$. Furthermore, the poles of det $\hat{D}(s)^{-1}$ are $j(2 \pi / L) k, k=0, \pm 1, \pm 2, \cdots$, and they lie entirely on the imaginary axis. Hence, $\hat{D}(s)$ is purely unstable. Therefore, we can apply the results in the previous section to a servo problem with periodic exogeneous signals. It is to be noted here that the approach by Francis [10], and Desoer and Gustafson [8] cannot be adopted to this problem, since $\hat{D}(s)^{-1}=(\exp (L s)-1)^{-1}$ admits no pseudocoprime factorizations nor does it satisfy the hypotheses of [8] because of the infinitely many poles on the imaginary axis.

Theorem 4.10 asserts that the model $(\exp (L s)-1)^{-1}$ must be contained in the forward path of the closed-loop system for stable tracking to periodic reference commands. Conversely, we know from Theorem 4.10 that internal stability of the closed-loop system together with the internal model $(\exp (L s)-1)^{-1}$ yields tracking, i.e., exponential decay of the error signal. (By abusing the terminology, we shall also call the transfer function of the model an internal model as well as its denominator.) The sufficiency was first pointed out by Inoue et al. [16] for SISO systems and by Hara et al. [13] for the MIMO case, while the necessity of the internal model has not been proved. The servo system with the internal model $(\exp (L s)-1)^{-1}$ is called a repetitive control system. However, it is a nontrivial problem to stabilize the repetitive control system, since the system under consideration turns out to be a delay-differential system of neutral type. Thus, repetitive control systems have been studied mainly on analysis and synthesis of stabilizing controllers [13]-[15]

The remainder of this section is devoted to the stability analysis. For notational simplicity, only the SISO case is considered, since the analogous discussions carry over to the MIMO case.

Let us now consider a repetitive control system whose openloop system consists of the internal model $(\exp (L s)-1)^{-1}$ and a finite-dimensional linear system $g(s)=\beta(s) / \alpha(s)$, where $\alpha(s)$ $=\alpha_{n} s^{n}+\cdots+\alpha_{1} s+\alpha_{0}$ and $\beta(s):=\beta_{n} s^{n}+\cdots+\beta_{1} s+\beta_{0}$ have no common factors and $\alpha_{n} \neq 0$ (see Fig. 1). Let us take the following factorization:

$$
\hat{Q}(s)=(\exp (L s)-1) \alpha(s), \hat{P}(s)=\beta(s) .
$$

It is then immediate from Theorems 3.12 and 4.10 that if

$$
\hat{q}_{o}(s)=(\exp (L s)-1) \alpha(s)+\beta(s)
$$

satisfies (3.13), then the error $e(t)$ tends exponentially to zero for any periodic reference signal $r(t)$ with period $L$. A sufficient condition for this is given as follows.

Lemma 5.5: Under the assumptions above, if

i) $g(s)=\beta(s) / \alpha(s)$ is proper stable, and

ii) $\|1-g\|_{\infty}<1$

then $\hat{q}_{o}(s)$ defined by (5.4) satisfies (3.13).

Proof. Rewrite $\hat{q}_{o}(s)$ as

$$
\hat{q}_{o}(s)=\alpha(s) \cdot\{\exp (L s)-(1-g(s)\} .
$$

Condition i) implies $\alpha(s)^{-1} \in H^{\infty}$. Since $|\exp (L s)| \geq 1$ for $\operatorname{Re} s$ $\geq 0$, condition ii) guarantees that $\{\exp (L s)-(1-g(s))\}^{-1}$ belongs to $H^{\infty}$. Hence, condition i) in (3.13) is satisfied. Since ord $q=n$, the proof will be complete if we show

$$
\sup _{\omega}\left|\frac{\omega^{n} / \alpha(j \omega)}{\exp (j \omega L)-(1-g(j \omega))}\right|<\infty .
$$

Since the denominator is bounded away from 0 by condition ii) in (5.6) and $\sup \left|\omega^{n} / \alpha(j \omega)\right|<\infty$, (5.8) follows.

Summarizing the above discussions, we have the following theorem.

Theorem 5.9: Consider a repetitive control system with

$$
\hat{Q}(s)=(\exp (L s)-1) \alpha(s), \hat{P}(s)=\beta(s)
$$

in Fig. 2, where $\alpha(s)$ and $\beta(s)$ are polynomials with no common factors. If $g(s)=\beta(s) / \alpha(s)$ satisfies condition (5.6), then the error $e(t)$ tends exponentially to zero for any periodic reference signal $r(t)$ with period $L$.

The above result corresponds to $[15$, Theorem 2] and [13, Theorem 1] where only the convergence in the sense of $L^{2}$ is assured in [13]. An entirely parallel discussion can be carried out for the case when the internal model included is expressed as (exp $(L s)-1)^{-1}+a(s)$, which has been investigated in [13] and [15]. For example, in the case of $a(s)=1$, we obtain

$$
\hat{Q}(s)=(\exp (L s)-1) \alpha(s), \hat{P}(s)=\exp (L s) \beta(s) .
$$

Hence, under the same assumption of Theorem 5.9, if $g(s)=$ $\beta(s) / \alpha(s)$ satisfies

$$
\begin{aligned}
& \text { i) }(1+g(s))^{-1} \text { is proper stable, and } \\
& \text { ii) }\left\|(1+g)^{-1}\right\|_{\infty}<1 \text {, }
\end{aligned}
$$

then the error e(t) tends exponentially to zero.

Note here that the second condition in $(5.6)$ or $(5.11)$ can be 
satisfied only when $g(s)$ is not strictly proper, i.e., $\beta_{n} \neq 0$. We will now show that this condition is actually necessary for internal stability. According to Theorem 3.9, the closed-loop transfer function $\hat{q}_{o}(s)^{-1}$ must belong to $H^{\infty}$ for the internal stability. Suppose that $\beta_{n}=0$, that is, $g(s)=\beta(s) / \alpha(s)$ is strictly proper. In view of $(5.4), \hat{q}_{o}(s)=\alpha(s) \cdot\{(\exp (L s)-1)+g(s)\}$. Since $g(s)$ is strictly proper, $g(j \omega) \rightarrow 0$ as $|\omega| \rightarrow \infty$. An easy application of Rouché's theorem [19] then shows that $(\exp (L s)-$ 1) $+g(s)$ has zeros close to $s= \pm 2 n \pi j / L$, for all sufficiently large $n$, and these zeros asymptotically tend to the imaginary axis. Thus, $\hat{q}_{o}(s)$ possesses a sequence of zeros with the same property, and this contradicts the requirement $\hat{q}_{o}(s)^{-1} \in H^{\infty}$. Hence, $\beta_{n}$ must be nonzero.

Thus, we have the following theorem on necessity which has not been clarified in the literature.

Theorem 5.12: Consider the SISO unity feedback system Fig. 2. If the closed-loop system is internally stable and the error $e(t)$ tends exponentially to zero for any periodic signal $r(t)$ with period $L$, then $i)(\exp (L s)-1)^{-1}$ must be contained in the forward path of the closed-loop system. Furthermore, under the same assumption in Theorem 5.9, ii) $g(s)$ is bicausal, i.e., $\beta_{n} \neq 0$.

Once we have arrived at the basic configuration as Fig. 1, it is also possible to discuss its stability via the stability results for neutral systems (see, e.g., [12]); for example, if the plant satisfies (5.6), then the spectrum of the closed-loop system is contained in the left-half plane $\{z ; \operatorname{Re} z<-c\}$ for some $c>0$, hence stable. However, we emphasize here that the necessity of an internal model for this class can be first proved via the use of the present method and it is difficult to conceive the configuration of Fig. 1 without the internal model principle as above (or Theorems 4.7 or 4.10).

Finally, a numerical example is shown to illustrate the results. We consider a repetitive control system consisting of a bicausal finite-dimensional system

$$
g(s)=\frac{5\left(s^{2}+3 s+3\right)}{2\left(s^{2}+3 s+1\right)}
$$

and the internal model

$$
(\exp (L s)-1)^{-1}+1=\exp (L s) /\{\exp (L s)-1\} .
$$

Since $g(s)$ satisfies the conditions (5.11), the error $e(t)$ tends exponentially to zero for any periodic signal with period $L$ in this repetitive control system. This is shown by a simulation result Fig. 4, where the reference command $r(t)$ has a triangular type waveform with period 4 , and $y(t)$ and $u(t)$ denote the controlled output and the output of the internal model (5.14), respectively. Observe that the tracking error is reduced to a very low level after the fifth period.

In contrast to this satisfactory result, stable tracking cannot be achieved for the strictly proper plant case by Theorem 5.12, which is illustrated by Fig. 5, where

$$
g(s)=\frac{15(s+1)}{2\left(s^{2}+3 s+1\right)}
$$

and the internal model given by (5.14). Although the tracking error may tend to zero, the internal stability cannot be assured, since $u(t)$ becomes larger in amplitude and contains higher frequency oscillations as $t$ goes to infinity. We note that introduction of an approximate internal model for the periodic signal generator improves the stability margin and enables us to achieve satisfactory high accuracy tracking for strictly proper systems. This modification has been adopted in many practical applications [13], [16], [17]. An approximate internal model can be given as

$$
\frac{\exp (L s)}{\exp (L s)-g_{f}(s)}+a(s)
$$
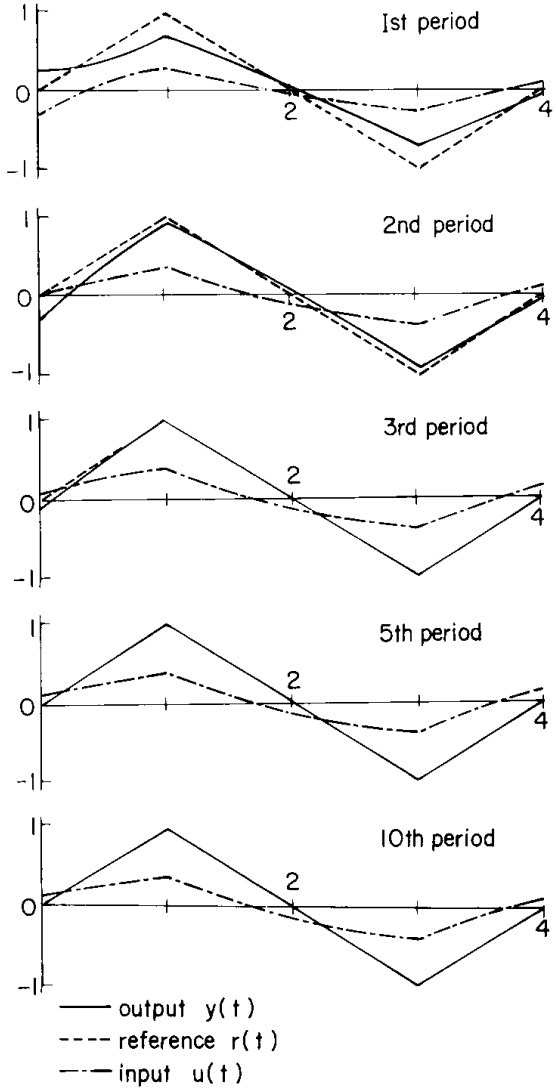

Fig. 4. Simulation: plant with direct path.

where $a(s)$ and $g_{f}(s)$ are proper stable rational functions and $g_{f}(s)$ has low-pass and high-cut characteristics [13], [15]. The effect of this modification is illustrated in Fig. 6, where $g(s)$ is given by (5.15) and the approximate internal model is of the form (5.16) with $a(s)=1$ and $g_{f}(s)=1 /(1+0.01 s)$. We see that the internal stability is assured together with the property of steadystate tracking error near the peaks of the reference command, which include much higher frequency components.

\section{Concluding Remarks}

In this paper, we have derived various necessary and sufficient conditions for internal and external stability, and applied the results to the study of an infinite-dimensional servo problem.

The results obtained are roughly summarized as follows.

i) Internal $L^{2}$-stability and exponential stability are equivalent.

ii) If the system is internally stable, then its transfer function matrix belongs to $H^{\infty}$.

iii) If the denominator of the transfer function satisfies a stronger condition (3.13), then the system $\Sigma Q$ is internally stable; in particular, this gives a sufficient condition for the internal stability of the canonical realization of a pseudorational impulse response under the condition that its fractional representation is approximately left coprime.

iv) An internal model of the exogenous signal is necessary for asymptotic tracking; it is also sufficient for tracking under the condition of the closed-loop internal stability; these results, along with the stability conditions obtained in Section III, have been effectively utilized for the analysis of repetitive control systems.

As a result of studying internal stability, we have naturally obtained exponential decay of the error $e(t)$ in the servo problem considered in Sections IV and V, in contrast to the existing results 

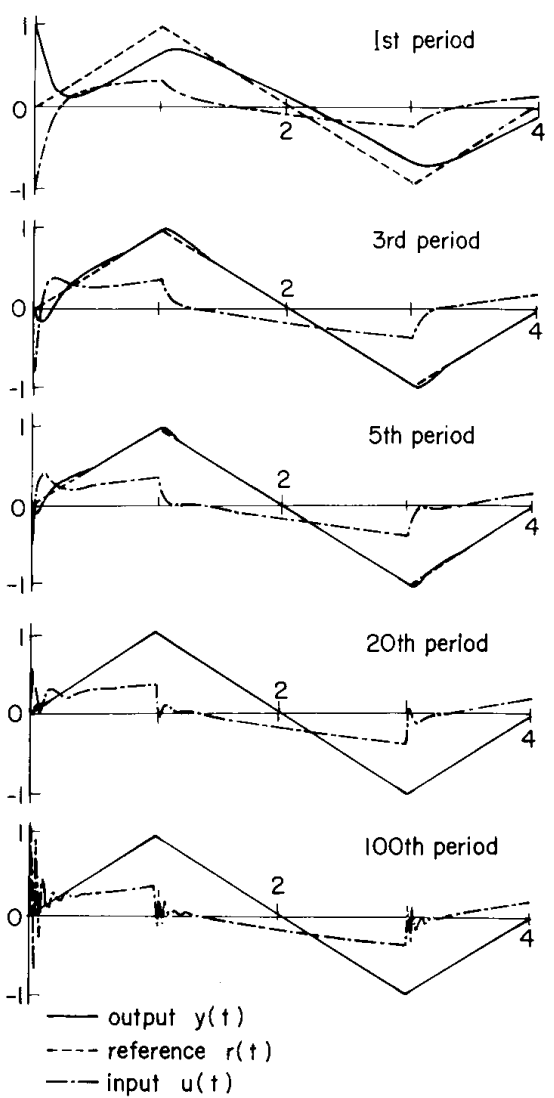

Fig. 5. Simulation: plant of relative degreee one. The case without a lowpass filter.

given in terms of external stability. Note also that iii) shows that, under suitable conditions, we can obtain an internally stable canonical realization if its transfer function satisfies a strong external stability condition (3.13).

\section{APPENDIX}

Proof of Theorem 3.9

Since the internal stability of $\Sigma^{Q, P}$ implies that of $\Sigma^{Q, P_{1}}$, the second statement is immediate from the first. To prove the first statement, we claim that there exists a constant $C>0$ such that

$$
\|A * u\|_{2} \leq C\|u\|_{2}
$$

for every $u \in\left(L^{2}(-\infty, \infty)\right)^{m}$. To this end, let us first prove that

$$
\|\pi(A * u)\|_{2} \leq C\|u\|_{2}
$$

for every $u \in \Omega^{m}$. In fact, since the correspondence $u \mapsto \pi(A * u)$ is continuous as a mapping from $\Omega^{m}$ to $\left(L_{\mathrm{loc}}^{2}[0, \infty)\right)^{p}$, we have

$$
\|\pi(A * u)\|_{[0, T]} \leq C_{0}\|u\|_{[-T, 0]}, u \in\left(L^{2}[-T, 0]\right)^{m}
$$

where $T>0$ is any real number for which $\|\cdot\|_{[0, n]}$ serves as a norm of the space $X^{Q}$. Observe that $\|\pi(A * u)\|_{\left[k T,(k+1) T_{1}\right.}=$ $\left\|\sigma_{k T}(\pi(A * u))\right\|_{[0, T]}$. Then by the exponential stability, we have

$\|\pi(A * u)\|_{[k T,(k+1) T]} \leq C_{1} \exp (-\beta k T)\|\pi(A * u)\|_{[-T, 0}$
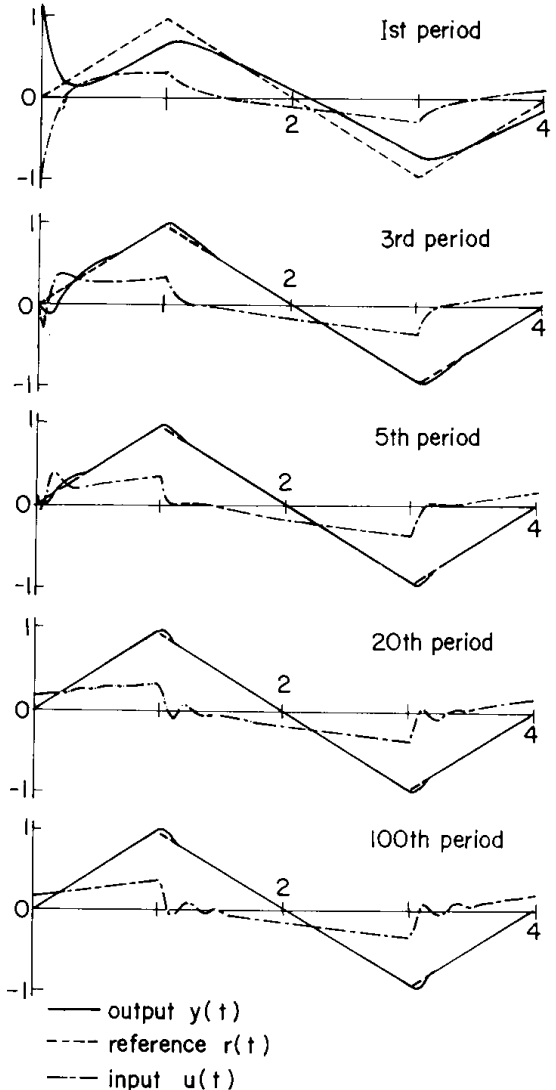

Fig. 6. Simulation: plant of relative degree one. The case with a low-pass filter.

for some $\beta>0$. This yields

$\|\pi(A * u)\|_{2} \leq C_{2}\left\{\sum_{k=0}^{\infty} \exp (-\beta k T)\right\}\|u\|_{[-T, 0]} \leq M\|u\|_{[-T, 0]}$.

Now take any $u \in \Omega^{m}$, and express it as

$$
u=\sum_{k=0}^{n} \sigma_{k T} u_{k}, u_{k} \in\left(L^{2}[-T, 0]\right)^{m}
$$

Then, again by the exponential stability and by (A.5), we have

$$
\begin{aligned}
\|\pi(A * u)\|_{2} & =\left\|\Sigma \sigma_{k T} \pi\left(A * u_{k}\right)\right\|_{2} \\
& \leq C_{3} \Sigma \exp (-\beta k T)\left\|\pi\left(A * u_{k}\right)\right\|_{2} \\
& \leq C_{4} \Sigma \exp (-\beta k T)\left\|u_{k}\right\|_{[-T, 0]} \\
& \leq C_{4}\{\Sigma \exp (-\beta k T)\}\|u\|_{2} .
\end{aligned}
$$

Thus, (A.2) holds. Let us next estimate the $L^{2}$-norm of $A * u-$ $\pi(A * u)$ which has support contained in $(-\infty, 0]$. First note that

$$
\|A * u\|_{[-T, 0]} \leq M_{1}\|u\|_{[-T, 0]}, u \in\left(L^{2}[-T, 0]\right)^{m} .
$$

This is valid since $A$ is a measure. Now take $u$ as in (A.6). It 
1052

follows that

$$
\begin{array}{rl}
\| A & * u \|_{[-(i+1) T,-i T]} \\
& \leq \Sigma_{k=i}\left\|A *\left(\sigma_{k T} u_{k}\right)\right\|_{[-(i+1) T,-i T]} \\
& =\Sigma_{k=i}\left\|A *\left(\sigma_{(k-i) T}\left(\sigma_{i T} u_{k}\right)\right)\right\|_{[-(i+1) T,-i T]} \\
& =\Sigma_{m=0}\left\|A *\left(\sigma_{m T}\left(\sigma_{i T} u_{m+i}\right)\right)\right\|_{[-(i+1) T,-i T]} \\
& =\left\|A * u_{i}\right\|_{[-T, 0]}+\Sigma_{k=0}\left\|A *\left(\sigma_{k T} u_{k+i}\right)\right\|_{[0, T]} \\
& \leq M_{1}\left\|u_{i}\right\|_{[-T, 0]}+\Sigma_{k=0} C_{0} \exp (-\beta k T)\left\|u_{k+i}\right\|_{[-T, 0]} \\
& \leq \Sigma_{k=0} M_{1} \exp (-\beta k T)\left\|u_{k+i}\right\|_{[-T, 0]} .
\end{array}
$$

Therefore, we have

$$
\begin{aligned}
& \left\{\|A * u\|_{[-(i+1) T,-i T]}\right\}^{2} \leq \Sigma_{j, k=0} M_{1}^{2} \\
& \cdot \exp (-\beta(k+j) T)\left\|u_{j+i}\right\|_{[-T, 0]} \cdot\left\|u_{k+i}\right\|_{\{-T, 0]} .
\end{aligned}
$$

It now follows that

$$
\begin{array}{rl}
\| A & * u-\pi(A * u) \|_{2}^{2} \\
= & \Sigma_{i=0}\left\{\|A * u\|_{[-(i+1) T,-i T]}\right\}^{2} \\
\leq & \Sigma_{i=0}\left\{\Sigma_{j, k=0} M_{1}^{2} \exp (-\beta(k+j) T)\left\|u_{j+i}\right\|_{[-T, 0]}\right. \\
& \left.\cdot\left\|u_{k+i}\right\|_{[-T, 0]}\right\} \\
= & \Sigma_{j, k=0} M_{1}^{2} \exp (-\beta(k+j) T)\left\{\Sigma_{i=0}\left\|u_{j+i}\right\|_{[-T, 0]}\right. \\
& \left.\cdot\left\|u_{k+i}\right\|_{[-T, 0]}\right\} \\
\leq & \Sigma_{j, k=0} M_{1}^{2} \exp (-\beta(k+j) T) \cdot\left\{\Sigma_{i=0}\left\|u_{j+i}\right\|_{[-T, 0]^{2}}\right\}^{1 / 2} \\
& \cdot\left\{\Sigma_{i=0}\left\|u_{k+i}\right\|_{\left.[-T, 0]^{2}\right]^{1 / 2}}\right. \\
\leq & \Sigma_{j, k=0} M_{1}^{2} \exp (-\beta(k+j) T) \cdot\|u\|_{2}^{2} \\
\leq & C_{5}\|u\|_{2}^{2} .
\end{array}
$$

This last estimate does not depend on the number $n$ of terms of $u$ in (A.6), so that it is valid as long as $u \in L^{2}(-\infty, 0]$. Combining this with (A.2), we obtain

$$
\|A * u\|_{2} \leq C\|u\|_{2}, u \in\left(L^{2}(-\infty, 0]\right)^{m} .
$$

Now in view of the shift invariance of the convolution $A * u$, (A.10) is valid for any $u \in\left(L^{2}(-\infty, k T]\right)^{m}$, and hence for any $u$ $\in\left(L^{2}(-\infty, \infty)\right)^{m}$. Thus, (A.1) is proved.

From this, it follows that the Laplace transform of $A$ belongs to $H^{\infty}$ (see, e.g., [23, Appendix]). Finally, the last statement follows from $\left\|Q^{-1}\right\|_{\infty}<\infty$.

\section{REFERENCES}

[1] G. Bengtsson, "Output regulation and internal models - A frequency domain approach," Automatica, vol. 13, pp. 333-345, 1977.

2] R. P. Boas, Jr., Entire Functions. New York: Academic, 1954.

[3] F. M. Callier and C. A. Desoer, "An algebra of transfer functions for distributed linear time-invariant systems," IEEE Trans. Circuits Syst., vol. CAS-25, pp. 651-662, 1978; correction IEEE Trans. Circuits Syst., vol. CAS-26, p. $360,1979$.

[4] C. M. Callier and J. Winkin, "Distributed system transfer functions of exponential order," Int. J. Contr., vol. 43, pp. 1353-1373, 1986.

[5] R. Datko, "Extending a theorem of A. M. Liapunov to Hilbert space," J. Math. Anal. Appl., vol. 32, pp. 610-616, 1970.
C. A. Desoer and M. Vidyasagar, Feedback Systems: Input-Output Properties. New York: Academic, 1975.

[7] C. A. Desoer and C. L. Gustafson, "Design of multivariable feedback systems with simple unstable plant," IEEE Trans. Automat. Contr., vol. AC-29, pp. 901-908, 1984

[8] - "Algebraic theory of linear multivariable feedback systems," IEEE Trans. Automat. Contr., vol. AC-29, pp. 909-917, 1984.

[9] W. F. Donoghue, Distributions and Fourier Transforms. New York: Academic, 1969.

[10] B. A. Francis, "The multivariable servomechanism problem from the input/output viewpoint," IEEE Trans. Automat. Contr., vol. AC22, pp. 322-328, 1977 .

[11] B. A. Francis and W. M. Wonham, "The internal model principle for linear multivariable regulators," J. Appl. Math. Optimiz., vol. 2, pp. $170-194,1975$.

[12] J. K. Hale, Theory of Functional Differential Equations. New York: Springer-Verlag, 1977

[13] S. Hara, T. Omata, and M. Nakano, "Synthesis of repetitive control systems and its applications," in Proc. 24th Conf. Decision Contr., 1985, pp. 1384-1392.

[14] S. Hara and Y. Yamamoto, "Stability of repetitive control systems," in Proc. 24th Conf. Decision Contr., 1985, pp. 326-327.

[15] S. Hara, Y. Yamamoto, T. Omata, and M. Nakano, "Repetitive control system: A new type servo system for periodic exogeneous signals," IEEE Trans. Automat. Contr., vol. 33, pp. 659-668, signals,

[16] T. Inoue, M. Nakano, T. Kubo, S. Matsumoto, and H. Baba, "High accuracy control of a proton synchrotron magnet power supply," in Proc. IFAC 8th World Congress, vol. XX, pp. 216-221, 1981.

[17] M. Nakano and S. Hara, "Microprocessor-based repetitive control," in Microprocessor-Based Control Systems, N. K. Shinha, Ed. Amsterdam, The Netherlands: Reidel, 1986, pp. 279-296.

[18] T. Omata, S. Hara, and M. Nakano, "Nonlinear repetitive control with application to trajectory control of manipulators," J. Robotic Syst. vol. 4 , no. 5 , pp. 631-632, 1987.

[19] W. Rudin, Real and Complex Analysis. New York: McGraw-Hill, 1974.

[20] L. Schwartz, Théorie des Distributions, 2me ed. New York: Hermann, 1966.

[21] L. Schwartz, Méthodes Mathématiques pour les Sciences Physiques. New York: Hermann, 1961.

[22] F. Treves, Topological Vector Spaces, Distributions and Kernels. New York: Academic, 1967.

[23] J. M. L. Valenca and C. J. Harris, "Nyquist criterion for input/output stability of multivariable systems," Int. J. Contr., vol. 31, pp. 917$935,1980$.

[24] M. Vidyasagar, H. Schneider, and B. A. Francis, "Algebraic and topological aspects of feedback stabilization," IEEE Trans. Automat. topological aspects of feedback stabilization.

[25] Y. Yamamoto, "Realization theory of infinite-dimensional linear systems, Parts I \& II," Math. Syst. Theory, vol. 15, pp. 55-77, 169190,1981 .

[26] - "Pseudorational input/output maps and their realizations: A fractional representation approach to infinite-dimensional systems," SIAM J. Contr. Optimiz., to be published; an earlier simplified version of this is available also as "On pseudo-rational linear input/
output maps," in Proc. IX IFAC World Congress, 1984, pp. 14691474 .

[27] $\frac{147 .}{}$, "A note on linear input/output maps of bounded-type," IEEE Trans. Automat. Contr., vol. AC-29, pp. 733-734, 1984.

[28] _ _ "Reachability of a class of finite-dimensional linear systems: An external approach with applications to general neutral systems, " SIAM J. Contr. Optimiz., to be published.

[29] Y. Yamamoto and S. Ueshima, "A new model for neutral delaydifferential systems," Int. J. Contr., vol. 43, pp. 465-472, 1986.

Yutaka Yamamoto (M'83), for a photograph and biography, see p. 667 of the July 1988 issue of this TRANSACTIONS.

Shinji Hara (M'87), for a photograph and biography, see p. 67 of the January 1988 issue of this TRANSACTIONS. 